\title{
Optimal problems in physical oceanography
}

\author{
R. D. Braddock
}

From the time a tsunami is generated until it arrives at a distant shore, it travels over large distances across the deep oceans. Although the functional relationship, linking the speed of the wave to the depth of the water through which it passes, has been known for many years, a method or technique for accurately plotting its full path has not been developed to date. Application of initial value techniques to the relevant equations has made possible only the plotting of the ray paths over short distances through shallow water.

Meteorological navigation or ship routing also demands the calculation of optimal paths across the oceans. Although some progress has been made in this field, the development of meteorological navigation has been retarded by lack of a method for calculating optimal paths.

A technique for calculating optimal paths has been developed, which can be used for the study of the propagation of a tsunami over an ocean as well as for the routing of shipping. This grid refinement (GR) technique is an iterative procedure which generates sequences of approximations to the travel time and to the optimal path between two fixed end points. Hence it is an end point procedure which can be used to solve the boundary value problems associated with the calculation of optimal paths.

Part I develops the GR-technique and uses the problem of the brachistochrone to investigate the effects of the various parameters associated with the method. The accuracy of the answers which are obtained is investigated, and it is shown that, under certain conditions, the technique provides a converging sequence of approximating paths. The relationship between the refinement parameters and the rate of convergence

Received 31 March 1969. Thesis submitted to the Flinders University of South Australia, August 1968. Degree approved, March 1969.

Supervisor: Professor J.R.M. Radok. 
of the sequence of approximating travel times is also discussed.

In Part II, the GR-technique is applied to several problems arising from the propagation of tsunamis in the Pacific Ocean. In some of these examples, a remarkable degree of correlation is obtained between the observed and the computed tsunami travel times. In other instances when the computed and observed valued disagree, in general, the inadequacy of the available depth data accounts for the discrepancies. The complex problems, which are associated with the propagation of the Alaskan tsunami of the 28th of March, 1964 in the South West Pacific, can be resolved and the tide gauge records of the event interpreted by use of the GR-technique.

The GR-technique has also been applied, with marked success, to a ship routing problem. The systematic collection and evaluation of the data, necessary for meteorological navigation, is still awaiting development. Hence only one example could be included.

Finally, in Part III, it is shown how the technique can be modified to handle more complex problems of the Calculus of Variations. Further possible applications of the GR-technique to other optimal problems, particularly meteorological navigation, are briefly discussed. The general character of the method suggests that it might find application in many branches of the physical sciences.

Thus, the GR-technique may meet the long felt demand for an effective method for the solution of the most important optimal problems of oceanography: tsunami propagation and ship routing. 